\title{
Neovasculature in 3D-PLGA/nHAp Scaffolds for Murine Critical Sized Bone Defect Regeneration by Photoacoustic Imaging: A Preliminary Study
}

\author{
Jian Li ${ }^{1}$, Bin Teng ${ }^{1}$, Ri-Qiang Lin ${ }^{2}$, Chang She ${ }^{3}$, Zhen-Yu Yao ${ }^{1}$, Jian Zhang ${ }^{4}$, Liang Song ${ }^{2}$, Pei-Gen Ren ${ }^{1}$ \\ Center for Translational Medicine Research and Development, Shenzhen Institutes of Advanced Technology, Chinese \\ Academy of Sciences, Shenzhen, Guangdong, China ${ }^{1}$ \\ Research Laboratory for Biomedical Optics and Molecular Imaging, Shenzhen Institutes of Advanced Technology, \\ Chinese Academy of Sciences, Shenzhen, Guangdong, China ${ }^{2}$ \\ Orthopedic Department, the Second Affiliated Hospital of Soochow University, Suzhou, Jiangsu, China ${ }^{3}$ \\ Laboratory for Reproductive Health, Shenzhen Institutes of Advanced Technology, Chinese Academy of Sciences, \\ Shenzhen, Guangdong, China ${ }^{4}$
}

\begin{abstract}
Reconstruction of large bone defects remains a challenge in the orthopaedic clinic. Genetic modification of biomaterial scaffold provides the opportunity to control the cellular microenvironment by inducing expression of tissue inductive factors to promote angiogenesis and osteogenesis. Angiogenesis in tissue-engineering scaffolds is essential for supplying oxygen and nutrients to the cells, removing waste products, and ultimately functionalizing implanted scaffolds. However, it was difficult to visualize and measure angiogenesis in three-dimensional (3D) scaffolds or new bone in bone tissue engineering in vivo and non-invasively. Photoacoustic microscopy (PAM) is a novel imaging modality that can acquire volumetric data in a non-invasive manner. In this study, we fabricated lentivirus-mediated genetic modification of 3D-PLGA/nHAp scaffold ( $\mathrm{PH})$, which can deliver recombinant lentivirus carrying cytokine gene-pdgfb (LV-pdgfb). In vitro, the modified scaffolds (PHp) continuously released bioactive LV-pdgfb particles for up to 5 days, and expressed PDGF-BB and significantly promoted migration of bone marrow-derived MSCs (BMSCs). In vivo, we detected that there were significant increasing of expressing of pdgfb and angiogenesis related genes. In this preliminary study, by using acoustic-resolution PAM (AR-PAM) and optical-resolution PAM (OR-PAM), we have investigated the blood vessels pattern in mouse calvaria in vivo. We have confirmed that PAM is a useful tool in evaluating neovasculature in bone tissue. In the future, we will quantify the neovasculature in 3D-scaffold which assisted bone regeneration by PAM scanning, and correlate the neovasculature with new bone regeneration in a murine calvarial critical bone defect model in the future work.
\end{abstract}

Keywords: critical bone defects, 3D scaffold, genetically modified neovascularization, photo acoustic imaging, bone regeneration.

\section{INTRODUCTION}

Bone is a dynamic and highly vascularized tissue within biodegradable materials, easy to be fabricated and the lifetime of an individual with a unique capacity to heal and remodel constantly[1]. It is generally believed that spontaneous bone regeneration is limited to relatively small defects, however, the large bone defects caused by traumatic injury, tumor removal, or genetic disorders and so on have to be restored by various grafts, for example, autografts, allografts, or artificial grafts made from biomaterials [2]. Autografts are considered the gold standard for the clinical therapies of critical-sized bone defects, but their use involves several problems, such as significant donor site morbidity, hemorrhage, deep infection, and chronic pain [3, 4].

Allografts are tissues obtained from genetically distinct sources such as human cadavers and donors, but their utilization is limited by the risks of pathogen transmission and immunological rejection $[5,6]$. Yet artificial grafts are promising alternatives, which can be made from

genetically modified $[7,8]$

Bone tissue engineering has emerged and offers new therapeutic strategies to aid musculoskeletal healing. Bone regeneration is a coordinated process that involves blood vessel in growth, different types of cells and growth factors [9]. To make bone regeneration more efficient, a wide variety of growth factors have been incorporated into scaffolds [10], such as bone morphogenetic proteins, vascular endothelial growth factor (VEGF) and fibroblast growth factor. Major barriers with protein therapy are cost, low bioavailability and supraphysiological dosage for therapeutic efficacy [11]. Therefore, to tackle these drawbacks, an alternative strategy to growth factor delivery for sustained bioactivity is gene therapy, which has been an effective approach for the treatment of osseous defects and diseases [12]. Platelet-derived growth factor-BB (PDGF-BB) known as potential chemoattractant 
and mitogen for mesenchymal and osteogenic cells and a stimulant for the expression of angiogenic molecules that play an important role in bone healing [13]. Inducing neovascularization formation in scaffold by PDGF-BB is one of the choices for enhancing tissue growing into scaffold in bone tissue engneering.

Osteogenesis and angiogenesis are two integrated components in bone repair and regeneration. Neovascularization in tissue-engineering scaffolds is essential for supplying oxygen and nutrients to the cells, removing waste products, and ultimately functionalizing implanted scaffolds. There are some imaging methods which can be used to obtain angiogenesis information instead of histomorphometry. Laser-scanning optical microscopy (LSM) including confocal and two-photon laser scanning microscopy, X-ray micro-computed tomography (microCT), scanning electron microscopy (SEM), optical coherence tomography (OCT), and magnetic resonance imaging (MRI) are commonly applied angiogenesis imaging methods.

However, there are various obstacles of these methods to visualize and measure neovasculature in 3D-scaffolds in bone tissue engineering. Recent rapid advances in photoacoustic microscopy (PAM) imaging techniques have made it possible to simultaneously visualize scaffolds, cells, and surrounding vascular networks in vivo in a noninvasive manner [14-16]. There are few studies about the dynamics of angiogenesis process in bone tissueengineering scaffolds by using intravital PAM, yet. In present study, we designed the lentivirus-mediated genetic modification of three-dimensional porous 3DPLGA/nHAp scaffolds (PLGA/nHAp/LV-pdgfb, PHp), by which to achieve better angiogenesis and bone regeneration for critical bone defect restoring. To verify whether pdgfb gene carried by the scaffolds could promote angiogenesis in porous 3D scaffolds in vivo, we utilized AR-PAM and OR-PAM to monitor neovascularization during bone formation in a murine calvarial critical bone defect model.

\section{MATERIALS AND METHODS}

\subsection{Materials}

Poly(D,L-lactide-co-glycolide) (PLGA) (L/G ratio 75:25, MW 66000-107000) and hydroxyapatite nanoparticles (nHAp average diameter $<200 \mathrm{~nm}$ ) were purchased from Sigma-Aldrich (St. Louis, MO, USA), and 1, 4-Dioxane purchased from Shanghai Lingfeng chemical reagent co., LTD. (Shanghai, China). Stericup filter unit was bought from Merck Millipore Corporation (Darmstadt, Germany). The cDNA of platelet derived growth factor BB (pdgfb) gene was purchased from Sino biological Inc. (Beijing, China). Anti-PDGF-BB mouse polyclonal antibody and PDGF-BB recombinant protein were from BioVision, Inc. (San Francisco, USA). Unless specified, all cell culture reagents were obtained from Life technology (NY, USA). The lentivirus vectors encoding GFP and Tomato were from Dr. Chiju Wei lab (Shantou University, China) and Dr. Sam S. Gambhir lab (Stanford University, USA), respectively.
2.2 Plasmid construct and lentivirus ( $\mathrm{LV}$ ) production The cDNA of pdgfb was cloned into lentiviral expression vector modified with a custom multiple-cloning site downstream of the cytomegalovirus promoter. Lentivirus was prepared in 293FT cells by transfection with the virus package plasmids using the standard calcium phosphate method [17]. After 48 hours of transfection, viral particles in culture supernatants were harvested and filtered with a Millipore Stericup filter unit (0.45 micron). Centrifugation was performed for $2 \mathrm{~h}$ at $25,000 \mathrm{rpm}$ unless otherwise stated. Supernatant was completely removed and virus pellets resuspended in $200 \mu \mathrm{l}$ PBS overnight at $4^{\circ} \mathrm{C}$ and stored at $-80^{\circ} \mathrm{C}$ until use. To determine the titer of lentivirus, HEK293T cells were incubated with serially diluted lentivirus solution for $24 \mathrm{~h}$, and the titer of the lentivirus was calculated as previously described [18].

2.3 Preparation of 3D PLGA/nHAp scaffolds and LV immobilization

Porous PLGA/nHAp scaffolds were fabricated by rapidprototyped method [19]. Briefly, PLGA material was dissolved in 1,4-dioxane to form a homogeneous solution and then nHAp powders was added to the solution with a PLGA:nHAp ratio of 10:1 (w/w). After that, the mixed solution was stirred vigorously using a magnetic stirrer to form a uniform paste. The material paste was fabricated into 3D scaffolds by a 3D low temperature printer (Tissue Form II, China) with computerized-nozzle to deposite layer-by-layer, bottom to top according to a predesigned model. And then, the scaffolds were vacuum freeze-dried to remove solvent remaining in the scaffolds as completely as possible. Then the scaffolds were soaked in $75 \%$ ethanol solution for 1 hour for sterilization and lyophilized again to get neutral, aseptic scaffolds. To obtain bioactive $\mathrm{LV}$ particle-coated scaffolds, $10 \mu \mathrm{LV}$ particles $\left(4.5 \times 10^{5}\right)$ were dropped onto each PLGA/nHAp scaffold, and scaffolds were incubated for 2 hours at $37^{\circ} \mathrm{C}$ in a humidified incubator to allow adsorption. After immobilization, the scaffolds were rinsed with PBS for twice to remove unbound LV particles. LV particle-coated scaffolds were snap-frozen in liquid nitrogen, lyophilized again, and stored at $-80^{\circ} \mathrm{C}$ for future use.

2.4 In vitro releasing kinetics of $\mathrm{LV}$ particles from scaffolds and LV transduction activity assay

Releasing of LV particles and the LV particles bioactivity studies from the scaffolds (dimensioned $4 \mathrm{~mm} \times 4 \mathrm{~mm} \times 2$ $\mathrm{mm}$ ) were performed. In brief, the $3 \mathrm{D}$ scaffolds carried LV-green fluorescent protein (LV-GFP, $4.5 \times 10^{5} \mathrm{LV}$ particles) were placed in $2.0 \mathrm{ml}$ cryogenic vials and incubated with $1 \mathrm{ml}$ complete DMEM medium at $37^{\circ} \mathrm{C}$ in a shaking incubator for 5 days. Sampling and replacement of the releasing medium was carried out every 12 hours starting from 12 hours to 120 hours post-incubation. At each time point, the whole sample volume was taken out and replaced with $1 \mathrm{ml}$ fresh complete DMEM medium. The collected media were used for transducing HEK293T cells by co-incubating for 48 hours. At 2 days after transduction, the number of bioactive LV particles was measured by flow cytometry to calculate the number of GFP-positive HEK293T cells. 
2.5 In vitro migration studies with bone marrow-derived MSCs

The migration assay of BMSCs was performed with a Boyden chamber, using a 24-well plate and polycarbonate filters (Transwell Costar, Corning, Acton, MA, USA) with a pore size of $8 \mu \mathrm{m}$. Prior to the assay, BMSCs expressing tomato fluorescent protein (BMSCs-T) or PDGF-BB protein (BMSCs-P) were prepared according to previous study [20], respectively. BMSCs-T $\left(5 \times 10^{4}\right)$ were placed in the upper chambers of Boyden transwell for migration. Based on what the contents in lower chambers, 6 groups were designed as following: A. Blank control, containing $500 \mu \mathrm{l}$ of serum free medium only; B. FBS control, containing $500 \mu \mathrm{l}$ of serum free medium supplemented with $10 \%$ FBS; C. PDGF-BB control, $500 \mu 1$ of serum free medium supplemented with $4 \mathrm{ng} / \mathrm{ml}$ PDGF-BB; D. PDGF$\mathrm{BB}$ seize group, containing $500 \mu \mathrm{l}$ of serum free medium supplemented with PDGF-BB (4 ng/ml) and anti-PDGF$\mathrm{BB}$ (4 ng/ml); E. BMSCs group, normal BMSCs $\left(2 \times 10^{5}\right)$ were placed in lower compartment containing $500 \mu \mathrm{l}$ of serum free medium; F. BMSCs-P group, BMSCs-P $\left(2 \times 10^{5}\right)$ were placed in lower compartment containing $500 \mu \mathrm{l}$ of serum free medium. After 48 hours incubation, migrated cells on the lower chamber side were removed and collected for quantification of the number of BMSCs-T by flow cytometry.

2.6 Establishment of mouse calvarial defect model and scaffold transplantation

Male BALB/c mice (7 weeks old) were purchased from Guangdong Medical Laboratory Animal Center (Guangdong, China) and all animals received care in compliance with the Guidelines outlined in the Guide for the Care and Use of Laboratory Animals. All the procedures were conducted using standard aseptic techniques in accordance with the protocols approved by the Ethics Committee for Animal Research, Shenzhen Institute of Advanced Technology, Chinese Academy of Sciences. A total of 42 male mice were enrolled in the calvarial bone defects experiment.

The animals were anaesthetized by intraperitoneal injection of pentobarbital sodium $(50 \mathrm{mg} / \mathrm{kg})$ during in the process of building critical bone defects model. $1 \mathrm{~cm}$ linear incision was made to reveal the bone surface and the periosteum was completely cleared from the surface of the cranium by scraping. A critical-size defect was created using a $4 \mathrm{~mm}$ diameter trephine burr with copious PBS irrigation and the full thickness of the calvarial bone was carefully removed [21]. The mice were randomly divided into three groups with fourteen in each group that received the following treatments: Group A, no implants; Group B, PH scaffolds implanted; and Group C, PHp scaffolds implanted. The skin wounds were sutured after the surgeries, and post-operational care was performed.

2.7 Gene expression analysis of pdgfb and angiogenesisrelated genes by RT-qPCR

To investigate the expression of pdgfb and the other two angiogenesis-related genes, RT-qPCR was performed. In the PH and PHp groups, implanted scaffolds $(n=4$ in each group) were harvested at 2,4 , and 8 weeks post- implantation; control group samples $(n=4)$, taken at the same time points, were from adjacent bone tissue. Total RNA was extracted from each sample using TRIzol reagent (Invitrogen, USA) according to the manufacturer's protocol. The RNA was then reverse transcribed to generate cDNA using the Reverse Transcription Kit (Takara, Japan) following the manufacturer's protocol. Quantitative real-time PCR was performed using the SYBR Green Detection System with a LightCyclerV ${ }^{\mathrm{R}} 480$ instrument (Roche, Switzerland). The sequences of the specific primers were as follow:

PDGF-BB forward primer: 5'-

CATCCGCTCCTTTGATGATCTT-3', PDGF-BB reverse primer: 5'-GTGCTCGGGTCATGTTCAAGT-3'; VWF forward primer: 5'-

CTCTTTGGGGACGACTTCATC-3', vWF reverse primer: 5'-TCCCGAGAATGGAGAAGGAAC-3';

VEGFR2 forward primer: 5'GAAATGACACTGGAGCCTACAAG-3', VEGFR2 reverse primer: 5'-TCCATGCTGGTCACTAACAGAAG3 ';

$\begin{array}{lcc}\beta \text {-actin } & \text { forward } & \text { primer: } \\ \text { GTATCCATGAAATAAGTGGTACAGG-3', } \beta \text {-actin }\end{array}$

reverse primer: $5^{\prime}$ -

GCAGTACATAATTTACACAGAAGCAAT-3'.

PCR was carried out with the following thermal conditions: $95^{\circ} \mathrm{C}$ for $30 \mathrm{~s}$ followed by 40 cycles of $95^{\circ} \mathrm{C}$ for $5 \mathrm{~s}$ and $60^{\circ} \mathrm{C}$ for $30 \mathrm{~s}$. Post PCR melting curves confirmed the specificity of single-target amplification, and the fold change of the gene of interest relative to $\beta$-actin was determined. The reaction for each sample was tested for at least three times.

2.8 AR-PAM system and imaging acquisition

The home-developed photoacoustic/ultrasound dualmodality imaging system (AR-PAM) was instrumented in Shenzhen Institutes of Advanced Technology, which consists of a tunable pulsed OPO laser (Vibrant 355 II HE, Opotek, Carlsbad, USA) that operated at $1064 \mathrm{~nm}$ for photoacoustic excitation, a focused ultrasound transducer (V315-SU, Olympus IMS, Waltham, USA; central frequency: $10 \mathrm{MHz}$; fractional bandwidth: $6 \mathrm{MHz}$; N.A.: 0.4) for both ultrasonic firing and photoacoustic/ultrasonic detection, and a precision motorized 3D scanning stage (PSA2000-11, Zolix, Beijing, China) to scan the imaging head across the $x-y$ plane for $3 \mathrm{D}$ imaging.

The laser beam area was measured $4 \mathrm{~mm} \times 4 \mathrm{~mm}$, which is large enough to uniformly illuminate the entire area of the scaffolds. One week after surgery, mice were anaesthetized by intraperitoneal injection of pentobarbital sodium $(50 \mathrm{mg} / \mathrm{kg}$ ) and immobilized on a head-fixing stage with a warming pad mounted to maintain body temperature throughout the experiments. Hydration was maintained by intraperitoneal injection of saline $(200 \mu \mathrm{l})$ at $1-2 \mathrm{~h}$ intervals. The $1.5 \%$ isoflurane gas (Keyuan, Shandong) in $100 \%$ oxygen was used to maintain the satisfactory anesthesia and analgesia during the imaging process. Volumetric data were collected and processed using custom MATLAB programs. 
2.9 OR-PAM system and imaging acquisition

The experimental setup for OR-PAM can be found in previous publications $[22,23]$. A 532-nm wavelength was from an Nd:YAG laser source (SPOT-532, Elforlight, UK), and the emitted laser beam with a pulse duration of around $1.8 \mathrm{~ns}$ was reshaped by a circular aperture of $2 \mathrm{~mm}$ in diameter. Then, a 75-MHz transducer (V2022, OlympusNDT, Japan) was used to detect the time-resolved photoacoustic signals from the sample. A-line pulse repetition rate of up to $5 \mathrm{kHz}$ was used in all experiments. The motor scanning and data acquisition were controlled by customized computer software written in LabView (2011, National Instruments). In vivo OR-PAM was performed followed by AR-PAM on the same animal prepared following the same procedure described in section 2.7.

\subsection{Statistical analysis}

For multiple comparisons, statistical significance of the differences among the groups was analyzed by one-way analysis of variance (ANOVA) with Tukey's Multiple Comparison Testing. A value of $\mathrm{p}<0.05$ was considered significant. Error bars represent the standard error in all figures. Prism (GraphPad) software was used for all data analysis.

\section{RESULTS}

3.1 Morphology of porous 3D PLGA/nHAp scaffolds Cylindrical Porous 3D PLGA/nHAp scaffolds $0.6 \mathrm{~mm}$ in height and $4 \mathrm{~mm}$ in diameter were fabricated with the 3D Tissue Form II printer. The morphology of the scaffolds were analyzed via scanning electron microscopy (SEM) and microCT, respectively. Fig. 1A showed the appearance of the implanted scaffold. Assessment of porosity of the scaffold by microCT found that more than $80 \%$ pores with a pore size ranging from 200 to $400 \mu \mathrm{m}$, which provided interconnectivity for cells ingrowth and an accurate cell distribution throughout the porous structure. High-resolution SEM imaging demonstrated that the surface of the scaffold had a rough microtopography, as seen in Fig. 1C. In addition, micropores with diameters of approximately 1-10 $\mu \mathrm{m}$ were also observed interconnected inside the scaffolds, which may help improve intercellular communication (Fig. 1D).

3.2 LV particle immobilization to scaffolds and released profile in vitro

Many studies have shown that virus can loss bioactivity when modified onto certain biomaterial carriers surfaces $[24,25]$. Thus it was important to test whether the LVGFP particles released from PLGA/nHAp scaffolds was active or not. We have determined that multiplicity of infection (MOI) of LV-GFP particles to HEK293T cells was 4 (Data not shown). During the first 24 hours, LVGFP particles burst released from 3D scaffolds with approximately $40 \%$ of the total amount of coated LV-GFP particles $\left(4.5 \times 10^{5}\right)$. The following 5 days, the LV particles were continually released, as in Fig. 2. These data demonstrated that most lyophilization immobilized LV particles in the scaffold were bioactive, and their bioactivity was maintained up to120 hours.
3.3 Expressed PDGF-BB as a potent chemotactic factor for BMSCs migration

The migration capacity of MSCs is influenced by a large range of growth factors, cytokines, and chemokines [26, 27]. To investigate whether expressed PDGF-BB as a potent chemotactic factor, in the context of inducing BMSCs migration, we observed BMSCs-T migration in transwells by FACS method (Fig. 3). Both the positive group (C) and the PDGF-BB-expressing group (F) all induced a significant level of BMSC migration compared with the other 4 groups, and migration of BMSCs in Group $\mathrm{F}$ was significantly higher than that in Group $\mathrm{C}$. There were no significant differences in BMSC migration among group A, B, D or E. These results suggested that the LV particles expressed bioactive PDGF-BB and effectively stimulated BMSCs migration

3.4 Analysis of genes expression

In order to compare the differences in gene expression profile among the three groups, the transcript expression levels of genes including pdgfb, vWF and VEGFR2 was quantified by RT-qPCR at 2, 4, and 8 weeks postimplantation, as shown in Fig. 4. As expected, pdgfb expression was dramatically increased in PHp group at all the three time points, but there was no difference in its expression between the control and $\mathrm{PH}$ groups from week 2 to week 8 (Fig. 4A). The results indicated that LV-pdgfb particles released from the scaffold and sucessfully transfected the local cells surrounding the bone defect and improved the mRNA expression, while there was no effect on endogenous pdgfb expression in the PH group. The mRNA expression levels of vWF and VEGFR2 in all three groups were gradually increasing and were highest in PHp group, but for the $\mathrm{PH}$ group, their expression was not significantly higher than that in the control group.

The mRNA expression of $\mathrm{VWF}$ and VEGFR2 was increased 8.9-fold in PHp group and 3.4-fold in $\mathrm{PH}$ group compared to control group at 8 weeks, respectively (Fig. $4 \mathrm{~B}, 4 \mathrm{C})$. These results revealed that genetically modified scaffolds significantly promoted angiogenesis marker genes expression in bone regeneration.

\subsection{Neovasculature monitored and quantified by PAM}

So far, it has been rarely reported to real-time track and quantify neovascular and bone defect healing in a 3D scaffold with relatively high spatial resolution. In order to evaluate the capability of neovascularization of scaffolds implanted in vivo, we have designed a home-developed photoacoustic microscopy imaging system to monitor angiogenesis within the bone defect area. Before the formal scanning experiment, firstly we obtained normal tissue components of mouse cranial area by AR-PAM/ORPAM systems. With or without skin, there was no significant difference for both conditions as shown in Fig. 5 and 6. In AR-PAM imaging, the using of easily accessible $1064 \mathrm{~nm}$ ns-pulsed Nd: YAG lasers not only reduce the cost of photoacoustic imaging, but also significantly reduce optical scattering for a possibly extended optical/photoacoustic imaging depth. As shown in Fig. 5, the ultrasound images are utilized to visualize bone anatomy (e.g., skin and skull edge), while the 


\section{International Advanced Research Journal in Science, Engineering and Technology}

Vol. 3, Issue 4, April 2016

photoacoustic images can reveal the distribution of blood depths as shown in Fig. 6. Overall, it can be seen that the vessels in the skull bone. Using OR-PAM imaging, first, high lateral resolution of OR-PAM has offered a unique we demonstrated a series of longitudinal images of the capacity to clearly identify the vasculature without any distribution of blood vessels in skull bone at different exogenous contrast agent in cranial bone.
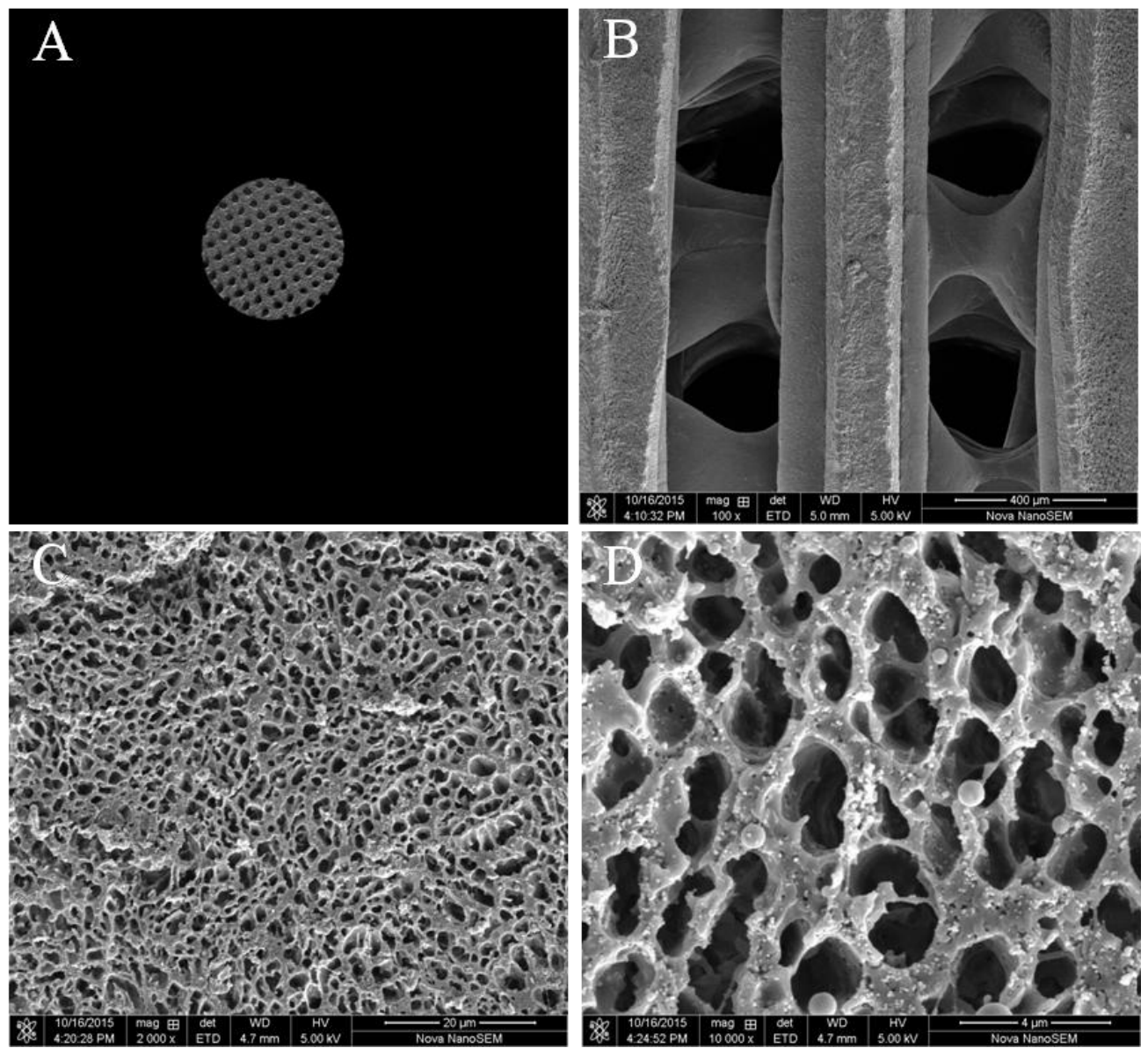

Fig.1. Morphology of porous 3D PLGA/nHAp scaffold. (A) The overall microporosity of the scaffold was analyzed by microCT, (B) SEM micrographs of the scaffold surface was observed at $100 \times, 2000 \times(C)$, and $10000 \times$ magnification (D).

\section{A}
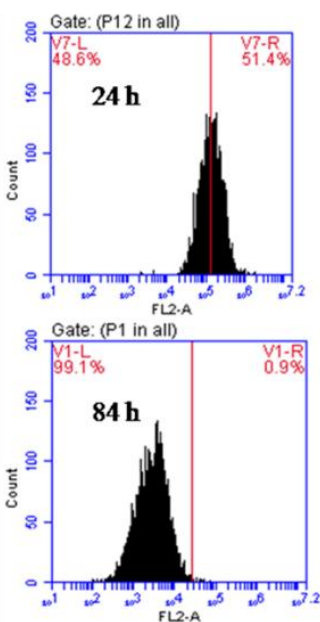
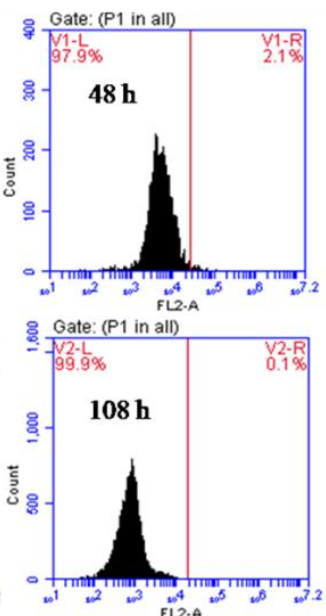

B

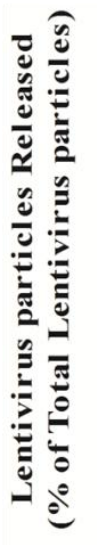

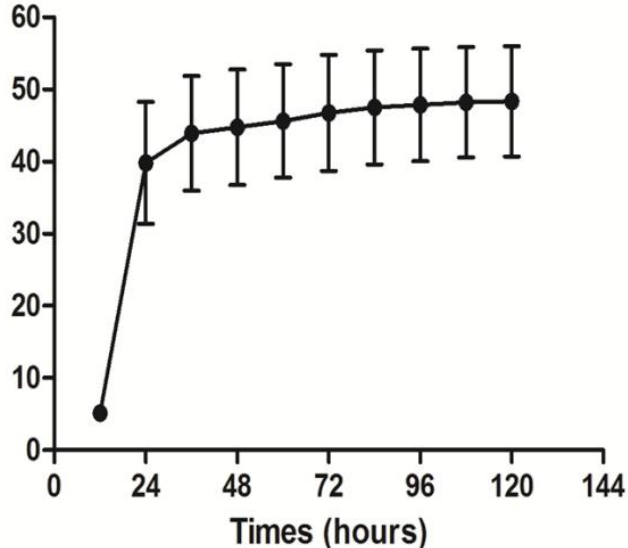

Fig.2. In vitro assessement of bioactivity of the LV-GFP particles by transfecting HEK293T cells via flow cytometry (A), and LV particles (LV-GFP) cumulative released from PLGA/nHAp scaffolds (B) at the given time points. All data are shown as mean \pm SEM $(n=4)$. 
International Advanced Research Journal in Science, Engineering and Technology

Vol. 3, Issue 4, April 2016

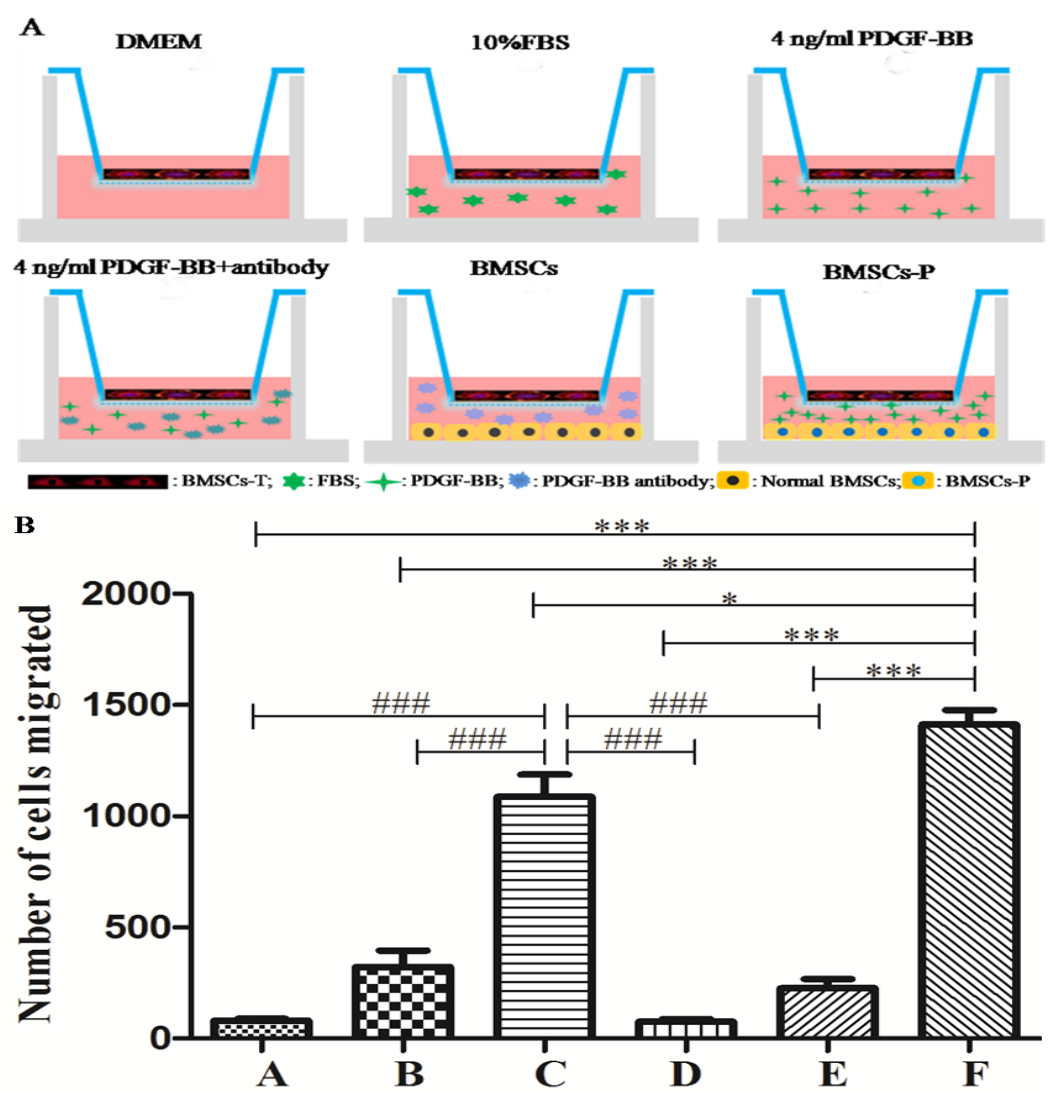

Fig.3. Quantitative measurement of cell migration in Boyden chambers. BMSCs-T $\left(5 \times 10^{4}\right)$ were seeded onto top of transwell chambers, with various condition mediums placed in the bottom of the chambers. All data are shown as mean \pm SEM. \#: comparison between group $\mathrm{C}$ and other groups, except group $\mathrm{F}$, \#\# $\mathrm{p}<0.0001$. *: comparison between group $\mathrm{F}$ and other groups, $* \mathrm{p}<0.05, * * \mathrm{p}<0.0001$.
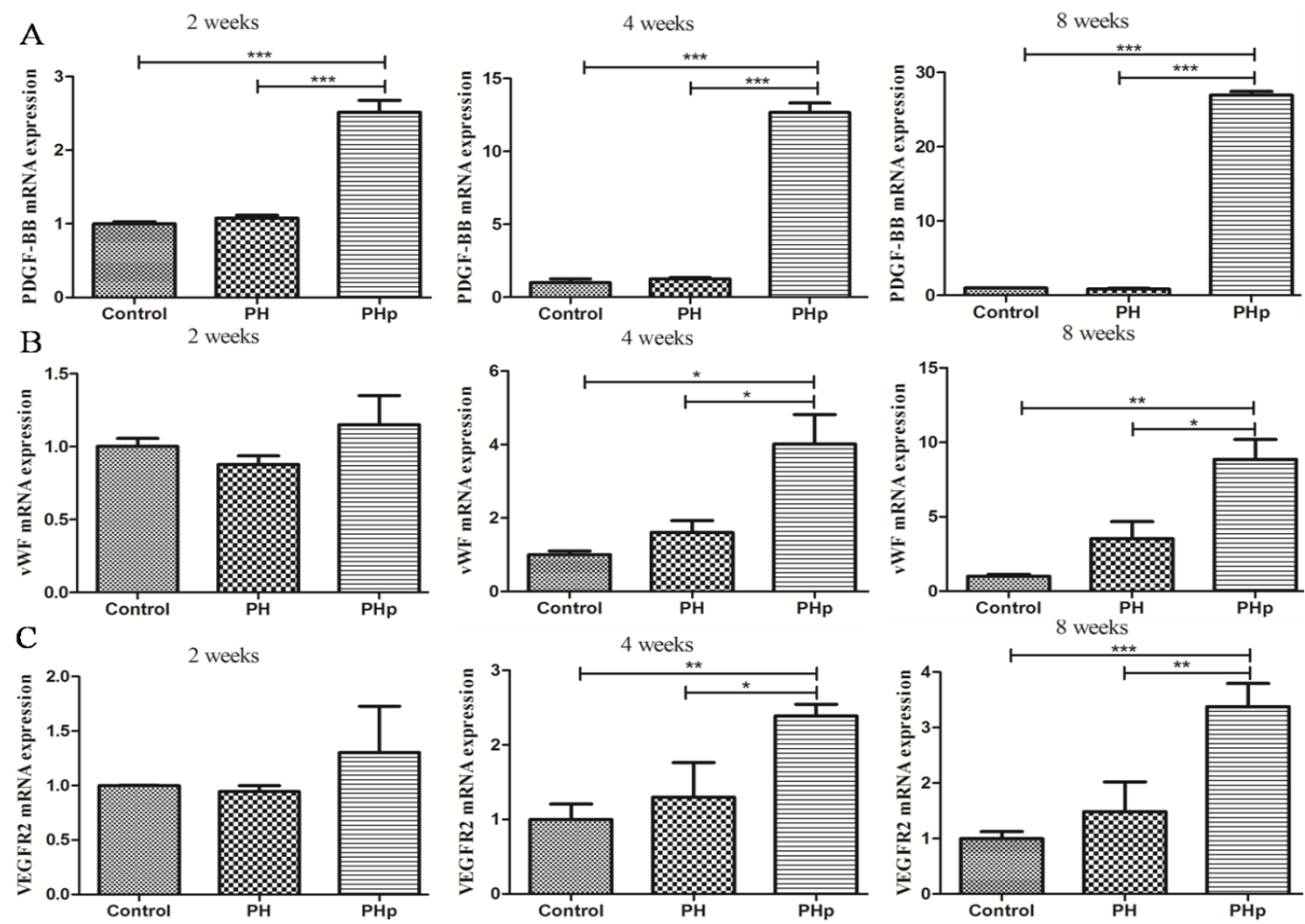

Fig.4. RT-qPCR analysis of genes expression in the three groups. (A) mRNA expression of PDGF-BB; (B) mRNA expression of vWF; (C) mRNA expression of VEGFR2. Samples from 4 animals were analyzed for each time point. ${ }^{* *} \mathrm{P}<0.05,{ }^{* *} \mathrm{P}<0.01$ and ${ }^{* * *} \mathrm{P}<0.0001$. 

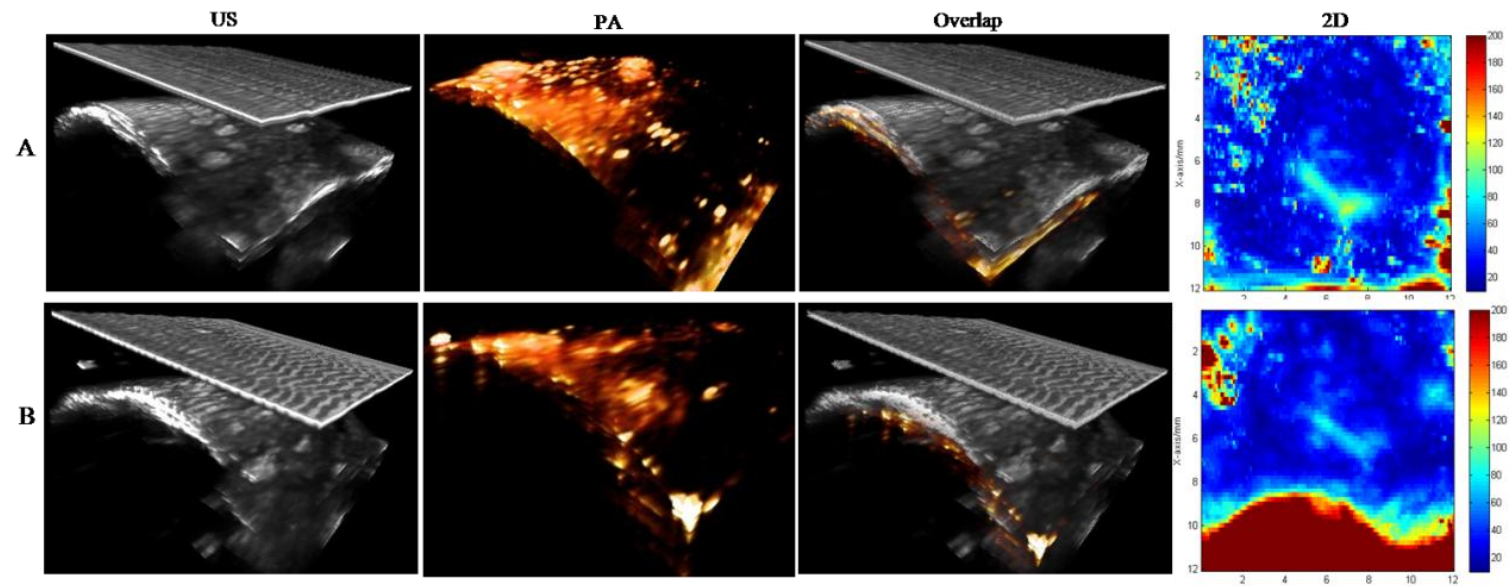

Fig.5. Ultrasound (US) and photoacoustic (PA) images along with their overlay images of normal tissue components of the cranial area with (A) or without skin (B) by AR-PAM.

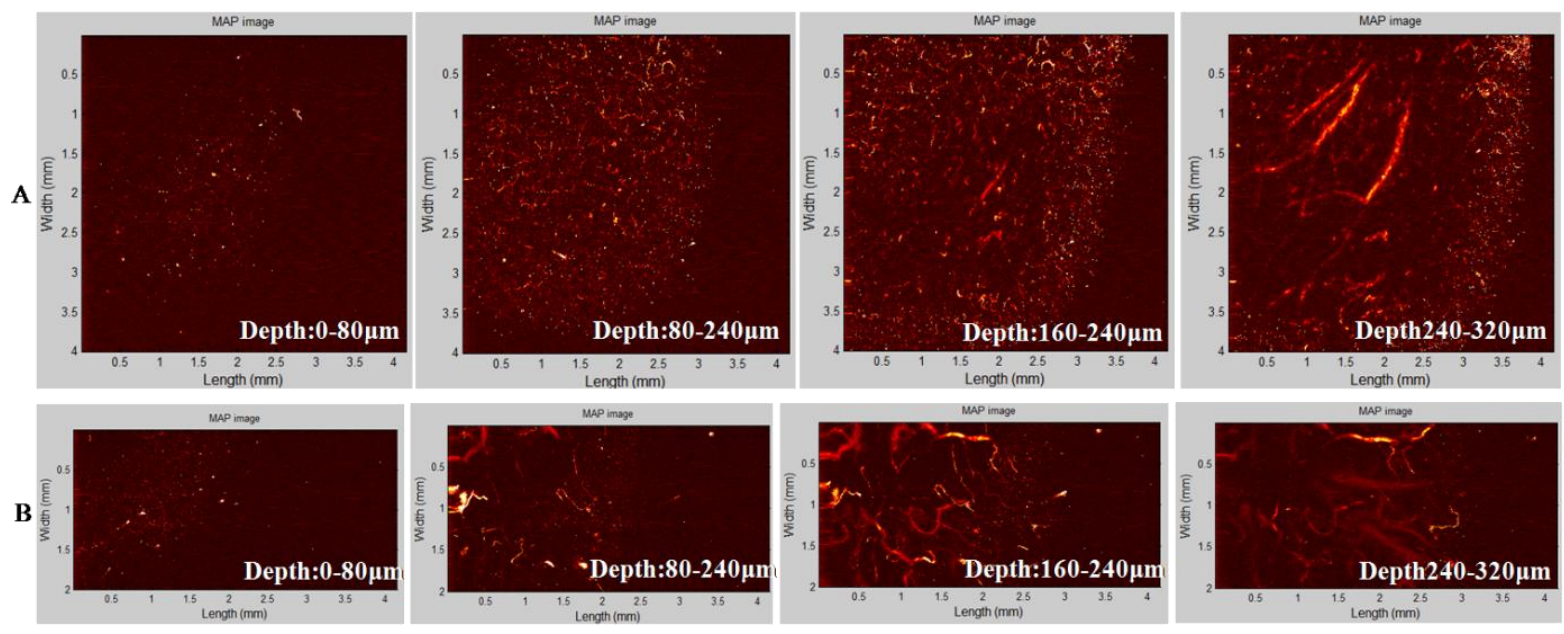

Fig.6. Imaging of normal tissue components of the cranial area with (A) or without skin (B) at different depths by ORPAM.

\section{DISCUSSION}

Bone is a dynamic and highly vascularized tissue. Bone formation is a complext process which requires synergetic functions of cells, growth factors, and scaffolds. However, the artificial delivery of proteinous growth factors may not supply a concerted working system to mimic the biological process, in the contrary, the improper dosage, stage or even toxicity might induce side effects. Expressible gene carried by scaffold can be an alternative to repair critical bone defects through a comprehensive, synergetic processing. Gene of growth factor like PDGF$\mathrm{BB}$ which can induce migration, differentiation, and proliferation of the BMSCs and osteoprogenitor cells could be taken in and expressed by adjacent cells after released from implanted scaffold. In the body, the whole repairing bio-system would work on its own according to the intrinsic biological mechanisms.

The level of osteogenesis is determined by the level of vascularization, and the low vascularization limits the wide clinical application of tissue-engineered bone. Constructing a highly vascularized of tissue-engineered bone according to the theory of biomimetics has become a useful method for repairing the large bone defect. In this study, we investigated whether pdgfb gene carried by the scaffolds could promote angiogenesis in porous 3D scaffolds in vivo, and whether photoacoustic imaging systems could monitor neovascularization during bone formation in a murine calvarial critical bone defect model. Various kinds of scaffolds have been successfully used for repairing of osseous defects. In order to achieve the aim of vascularization and osteogenesis, lentivirus-mediated genetic modification of PLGA/nHAp scaffolds were fabricated with controllable pore size and porosity. Neovascularization in tissue-engineering scaffolds is essential for supplying oxygen and nutrients to the cells, removing of waste products, and ultimate functionalization of the implanted scaffolds. Despite dramatic achievements in bone tissue engineering, visualizing and quantification neovascularization inside 3D-scaffolds is still a grand challenge. Quite rare microscopic imaging systems are capable of providing volumetric information of angiogenesis. Other currently used methods like micro-CT, MRI and histological analyses are destructive, complicated, laborious, or low spatial resolution and long image acquisition time [28]. 
Therefore, a non-invasive imaging modality with high resolution and penetration depth to provide volumetric information on angiogenesis in 3D tissue-engineering scaffolds will help to select and design more effective bone tissue engineering scaffolds for bone regeneration. PAM is a novel imaging modality that acquires volumetric data in a non-invasive manner [29]. It relies on photoacoustic signals generated by an optical absorbing species upon irradiation by a pulsed or intensitymodulated laser [30] and can detect both endogenous and exogenous [31, 32]. PAM works well on many scenarios of tissue engineering studies [14, 16, 28, 33]. It could provide high quality tomograph of big field of view of mouse skull as used in this study (Fig. 5 and 6). In addition, PAM can be used to measure the degradation profile of individual scaffolds, offering a new approach to non-invasively analyze and quantify biomaterial-tissue interactions in conjunction with the assessment of in vivo vascular parameters [34]. To our knowledge, there has no reports yet about the application of PAM on scaffoldbased bone tissue engineering.

PAM is expected to have broad applications in imaging 3D scaffold-based samples for regenerative medicine. Functional PAM can also be performed to derive other important parameters (e.g. oxygen saturation, flow velocity, oxygen partial pressure, and metabolicrate of oxygen) of the neovasculature. Next step, we will further optimize the imaging parameters of PAM in 3D scaffold implanted and utilize to real-time monitor neovascularization of scaffolds for $\mathrm{PH}$ and $\mathrm{PHp}$ groups in vivo by PAM imaging. However, PAM still has multiple technical challenges [14]. Thus, an integrated PAM system with a large range of switchable resolutions and penetration depths is of great interest. It is expected that further advancement of this fast-growing imaging method will find even broader applications for tissue engineering and regenerative medicine, especially when thick scaffold/tissue constructs are involved .

\section{CONCLUSION}

The objective of this preliminary study was to demonstrate the feasibility of combining AR-PAM and OR-PAM in a multi-modality approach to visualize and quantify the neovascularization inside 3D-scaffolds during bone formation in a murine calvarial critical bone defect model in bone tissue engineering and confirm whether pdgfbcontaining scaffolds (PHp) enhanced osteogenesis and angiogenesis for bone defect repair. The establishment of an intravital imaging platform will provide a unique tool to deeper understand of the cues of osteogenesis and angiogenesis and further facilitate the development of novel biomaterial-based therapeutics for improving defect repair and reconstruction.

\section{ACKNOWLEDGMENTS}

This work was supported by the following funds: 1 . Shenzhen Science and Technology Innovation Committee grants: JCYJ20130401170306839, JCYJ20120 615130350 920 and JCYJ20150401150223631; 2. Shenzhen Peacock Program grant: 110811003586331; 3. Guangdong public research and capacity building special grant: 2015A02021 2030; 4. The Second Affiliated Hospital of Soochow University Preponderant Clinic Discipline Group Project grant: XKQ2015003; 5. Suzhou Science and Technology Development Project grant: SYSD2015084.

\section{CONFLICT OF INTEREST}

The authors have declared that there is no conflict of interest regarding the publication of this paper.

\section{REFERENCES}

[1] Sommerfeldt DW, Rubin CT. Biology of bone and how it orchestrates the form and function of the skeleton. Eur Spine J. 2001;10:S86-S95

[2] Spin-Neto R, Landazuri Del Barrio RA, Pereira LA, Marcantonio RA, Marcantonio E, Marcantonio E, Jr. Clinical similarities and histological diversity comparing fresh frozen onlay bone blocks allografts and autografts in human maxillary reconstruction. Clinical implant dentistry and related research. 2013;15:490-7.

[3] Bertolai R, Catelani C, Aversa A, Rossi A, Giannini D, Bani D. Bone graft and mesenchimal stem cells: clinical observations and histological analysis. Clinical cases in mineral and bone metabolism : the official journal of the Italian Society of Osteoporosis, Mineral Metabolism, and Skeletal Diseases. 2015;12:183-7.

[4] Morrell NT, Weiss APC. Bone-Retinaculum-Bone Autografts for Scapholunate Interosseous Ligament Reconstruction. Hand Clin. 2015;31:451-+.

[5] Khan SN, Cammisa FP, Sandha HS, Diwan AD, Girardi FP, Lane JM. The biology of bone grafting. J Am Acad Orthop Sur. 2005;13:77-86.

[6] Park J, Chung WY. Osteochondral allograft reconstruction of talar body fracture with a large bone defect. Archives of orthopaedic and trauma surgery. 2015.

[7] Nie H, Ho ML, Wang CK, Wang CH, Fu YC. BMP-2 plasmid loaded PLGA/HAp composite scaffolds for treatment of bone defects in nude mice. Biomaterials. 2009;30:892-901.

[8] Elangovan S, D'Mello SR, Hong L, Ross RD, Allamargot C, Dawson DV, et al. The enhancement of bone regeneration by gene activated matrix encoding for platelet derived growth factor. Biomaterials. 2014;35:737-47.

[9] Barabaschi GD, Manoharan V, Li Q, Bertassoni LE. Engineering Pre-vascularized Scaffolds for Bone Regeneration. Advances in experimental medicine and biology. 2015;881:79-94.

[10] Zhang HX, Zhang XP, Xiao GY, Hou Y, Cheng L, Si M, et al. In vitro and in vivo evaluation of calcium phosphate composite scaffolds containing BMP-VEGF loaded PLGA microspheres for the treatment of avascular necrosis of the femoral head. Materials science \& engineering C, Materials for biological applications. 2016;60:298-307.

[11] Winn SR, Hu Y, Sfeir C, Hollinger JO. Gene therapy approaches for modulating bone regeneration. Advanced drug delivery reviews. 2000;42:121-38.

[12] Ramseier CA, Abramson ZR, Jin Q, Giannobile WV. Gene therapeutics for periodontal regenerative medicine. Dental clinics of North America. 2006;50:245-63, ix.

[13] Park SY, Kim KH, Shin SY, Koo KT, Lee YM, Seol YJ. Dual Delivery of rhPDGF-BB and Bone Marrow Mesenchymal Stromal Cells Expressing the BMP2 Gene Enhance Bone Formation in a Critical-Sized Defect Model. Tissue Eng Pt A. 2013;19:2495-505.

[14] Cai X, Zhang YS, Xia Y, Wang LV. Photoacoustic Microscopy in Tissue Engineering. Mater Today (Kidlington). 2013;16:67-77.

[15] Cai X, Paratala BS, Hu S, Sitharaman B, Wang LV. Multiscale photoacoustic microscopy of single-walled carbon nanotubeincorporated tissue engineering scaffolds. Tissue engineering Part C, Methods. 2012;18:310-7.

[16] Zhang Y, Cai X, Choi SW, Kim C, Wang LHV, Xia YN. Chronic label-free volumetric photoacoustic microscopy of melanoma cells in three-dimensional porous Scaffolds. Biomaterials. 2010;31:86518.

[17] Dull T, Zufferey R, Kelly M, Mandel RJ, Nguyen M, Trono D, et al. A third-generation lentivirus vector with a conditional packaging 
system. Journal of virology. 1998;72:8463-71.

[18] Cribbs AP, Kennedy A, Gregory B, Brennan FM. Simplified production and concentration of lentiviral vectors to achieve high transduction in primary human $\mathrm{T}$ cells. BMC biotechnology. 2013;13:98

[19] Zein I, Hutmacher DW, Tan KC, Teoh SH. Fused deposition modeling of novel scaffold architectures for tissue engineering applications. Biomaterials. 2002;23:1169-85.

[20] Gibon E, Batke B, Jawad MU, Fritton K, Rao A, Yao ZY, et al. MC3T3-E1 Osteoprogenitor Cells Systemically Migrate to a Bone Defect and Enhance Bone Healing. Tissue Eng Pt A. 2012;18:96873.

[21] Pigossi SC, de Oliveira GJ, Finoti LS, Nepomuceno R, Spolidorio LC, Rossa C, Jr., et al. Bacterial cellulose-hydroxyapatite composites with osteogenic growth peptide (OGP) or pentapeptide OGP on bone regeneration in critical-size calvarial defect model. Journal of biomedical materials research Part A. 2015.

[22] Yang Z, Chen J, Yao J, Lin R, Meng J, Liu C, et al. Multiparametric quantitative microvascular imaging with opticalresolution photoacoustic microscopy in vivo. Optics express. 2014;22:1500-11

[23] Lin R, Chen J, Wang H, Yan M, Zheng W, Song L. Longitudinal label-free optical-resolution photoacoustic microscopy of tumor angiogenesis in vivo. Quantitative imaging in medicine and surgery. 2015;5:23-9.

[24] Gersbach CA, Coyer SR, Le Doux JM, Garcia AJ. Biomaterialmediated retroviral gene transfer using self-assembled monolayers. Biomaterials. 2007;28:5121-7.

[25] Zhang Y, Ma Y, Wu C, Miron RJ, Cheng X. Platelet-derived growth factor BB gene-released scaffolds: biosynthesis and characterization. Journal of tissue engineering and regenerative medicine. 2013.

[26] Shen C, Lie P, Miao T, Yu M, Lu Q, Feng T, et al. Conditioned medium from umbilical cord mesenchymal stem cells induces migration and angiogenesis. Molecular medicine reports. 2015;12:20-30.

[27] Wu Y, Zhao RC. The role of chemokines in mesenchymal stem cell homing to myocardium. Stem cell reviews. 2012;8:243-50.

[28] Zhang Y, Cai X, Choi SW, Kim C, Wang LV, Xia Y. Chronic labelfree volumetric photoacoustic microscopy of melanoma cells in three-dimensional porous scaffolds. Biomaterials. 2010;31:8651-8.

[29] Zhang YS, Cai X, Yao JJ, Xing WX, Wang LHV, Xia YN. NonInvasive and In Situ Characterization of the Degradation of Biomaterial Scaffolds by Volumetric Photoacoustic Microscopy. Angew Chem Int Edit. 2014;53:184-8.

[30] Wang LV. Multiscale photoacoustic microscopy and computed tomography. Nat Photonics. 2009;3:503-9.

[31] Wang Y, Hu S, Maslov K, Zhang Y, Xia YN, Wang LV. In vivo integrated photoacoustic and confocal microscopy of hemoglobin oxygen saturation and oxygen partial pressure. Opt Lett. 2011;36:1029-31.

[32] Cho EC, Zhang Y, Cai X, Moran CM, Wang LHV, Xia YN. Quantitative Analysis of the Fate of Gold Nanocages In Vitro and In Vivo after Uptake by U87-MG Tumor Cells. Angew Chem Int Edit. 2013;52:1152-5.

[33] Cai X, Zhang Y, Li L, Choi SW, MacEwan MR, Yao JJ, et al. Investigation of Neovascularization in Three-Dimensional Porous Scaffolds In Vivo by a Combination of Multiscale Photoacoustic Microscopy and Optical Coherence Tomography. Tissue Eng Part C-Me. 2013;19:196-204.

[34] Zhang YS, Cai X, Yao J, Xing W, Wang LV, Xia Y. Non-invasive and in situ characterization of the degradation of biomaterial scaffolds by volumetric photoacoustic microscopy. Angewandte Chemie. 2014;53:184-8 